\title{
EXPLORATION OF VIDEO E-LEARNING CONTENT WITH SMARTPHONES
}

\author{
Stefan Räbiger, Tuğberk Dalkılıç, Alperen Doğan, Buket Karakaş, Berk Türetken \\ and Yücel Saygin \\ Faculty of Engineering and Natural Sciences, Sabancl University, Istanbul, Turkey
}

\begin{abstract}
Nowadays computer users prefer to learn or complement their studies with video materials. While there are many video e-learning resources available on the internet, video sharing platforms such as YouTube which provide these resources, do not structure the presented material in the prerequisite order. Furthermore, they do not track the background of the users when recommending the next material to watch. Our aim is to overcome this limitation of the existing video on demand systems. In this paper we describe the architecture of the e-learning system that we are developing which allows users to search and watch video materials organized with respect to their background and presented in prerequisite order. One of the key features of our e-learning platform is to enable users to explore the video content with mobile devices. We propose a new visual metaphor based on lists for mobile devices which reflect the prerequisite graph structure, utilizing the limited screen size more effectively.
\end{abstract}

\section{KEYWORDS}

e-Learning, Visualization, Mobile Learning, Visual Metaphor

\section{INTRODUCTION}

Nowadays students spend more time with mobile devices. Due to the high popularity of mobile devices, particularly smartphones, it is natural to provide learners access to learning material through mobile devices. We also observed that students tend to use video sharing platforms such as YouTube to find course supplementary materials or to learn new topics. However, video sharing systems are not designed to present video content with a coherent structure following the prerequisite order, also their personalization mechanisms are not tailored to take the academic background of their users into account when presenting material. For example, one can search on YouTube for a specific topic and a list of videos is returned in response to a query. As expected, the returned list of videos is good in terms of relevance with a lot of alternative materials. However, the user background is not considered by such systems except for the general interests identified by the previously watched videos. Also, the order of the recommendations by the video sharing platforms, suggesting what to watch next, does not follow the prerequisite order among the video content. In order to overcome these limitations of video sharing platforms, we are developing a new platform and a mobile app which acts as an interface to present the video learning materials in an order reflecting the prerequisite relationships. Our platform has a server component which contains a pool of concepts that we call micro-topics. The micro-topics form the learning space and they are organized in a graph structure with prerequisite relationships acting as a knowledge base. The mobile app component on the other hand acts as an interface for the user to search for video e-learning content for specific topics. The requested e-learning content is enriched with the prerequisites which are prefetched from the video sharing platform as a subgraph and displayed to the user on the mobile device. We have integrated the popular video sharing platform YouTube into our system; however, other video sharing platforms can also be used as the source for the video learning material.

In this paper, we describe the architecture of the general system and the mobile interface which maps the subgraph of video learning content with prerequisite information on a limited screen size. Screen size imposes constraints on the user interface since the screens of mobile devices are smaller than those of desktop computers and laptops. In our context this implies that the information conveyed by the graph must also be conveyed on mobile devices. To accomplish this goal, we propose a new visual metaphor based on lists which presents to 
learners only a small subset of the graph at a time, while still allowing learners to traverse the entire graph. This innovative list representation of the subgraph conveys the same information as the graph, utilizing the limited screen size more effectively, and allows easy interaction with the user to enable navigation in the graph through the mobile device. Initial user studies show that the mobile interface has the potential of being adopted by the students who would like to use existing resources in video sharing platforms.

\section{RELATED WORK}

The most relevant areas for our paper are existing e-learning platforms and how graphs are visualized on mobile devices. While the former one motivates the need for building a new e-learning platform, the latter one outlines why it is necessary to consider alternative metaphors for visualizing graphs on mobile devices with small screens.

\subsection{E-learning Platforms}

To the best of our knowledge, there are no other platforms which enable users to view existing learning material from popular video sharing platforms in an organized way. Existing systems are based on traditional courses and they transform these courses into massive online open courses where learners enroll in an online environment. Learners either register to those systems and enroll in the courses or they prefer to search general video sharing platforms for instructional videos. Our aim in this work is to enable learners to utilize the existing resources freely but in an organized way.

Popular e-learning platforms include Coursera, edX, and Udemy amongst others. However, these platforms adopt a "one size fits all" approach in the sense that a course is taught by displaying the same learning materials in the same order to different learners. This lack of content adaptation to individual learners is problematic since individuals learn differently. Splitting learning materials into smaller blocks (e.g. into micro-topics) is one method to adapt content to learners. This idea is commonly used in microlearning (Buchem \& Hamelmann, 2010), which was proposed as a new learning paradigm to teach new concepts to employees, who are only available for a limited amount of time at work. It is based on the idea of spaced learning, i.e. instead of teaching knowledge in one session, it is stretched over a longer period by providing materials that cover smaller topics, which has been shown to improve long-term memorization results of employees in randomized clinical trials (Cecilio-Fernandes et al., 2018). Various microlearning platforms exist, such as Auzmor (https://auzmor.com/learn/), Axonify (https://axonify.com/microlearning-platform/), Oplift (https://ocasta.com/oplift/products/engage), and Qstream (https://qstream.com), but they all neglect at least one of the following aspects: content adaptation, multiple learning materials per micro-topic, gamification, or content is provided by experts only. Furthermore, memorization does not imply a broader understanding of key concepts. And employees are the target audience of microlearning platforms which is reflected in their business models as accessing the content costs money. In contrast, our e-learning platform will combine the adaptive features of microlearning platforms and the openness of e-learning platforms. In addition, our platform will implement more methods to make the content adapt to learners such as giving them the freedom to choose the order in which they want to learn the different materials as long as this order does not violate any prerequisite relationships.

\subsection{Graph Visualizations on Mobile Devices}

Different methods exist to display graphs on mobile devices such as smartphones and tablets. On smartphones, which are our primary concern since learners tend to own smartphones instead of tablets, the authors of (Kister et al., 2017) combined the screens of multiple devices to display different regions of a graph. In our context this is not applicable since we cannot expect learners to possess multiple devices. In (Du et al., 2017), the authors evaluated different graph visualizations on single smartphones and noted that having to explore large parts of a graph increases the difficulty of completing the task as an extensive amount of panning and zooming interactions must be performed by users. This is also the reason why we propose an alternative visual metaphor based on lists. For tablets, on the other hand, it is feasible to provide visualizations that utilize a graph metaphor, e.g. (Drochtert \& Geiger, 2015). 


\section{ARCHITECTURE AND IMPLEMENTATION}

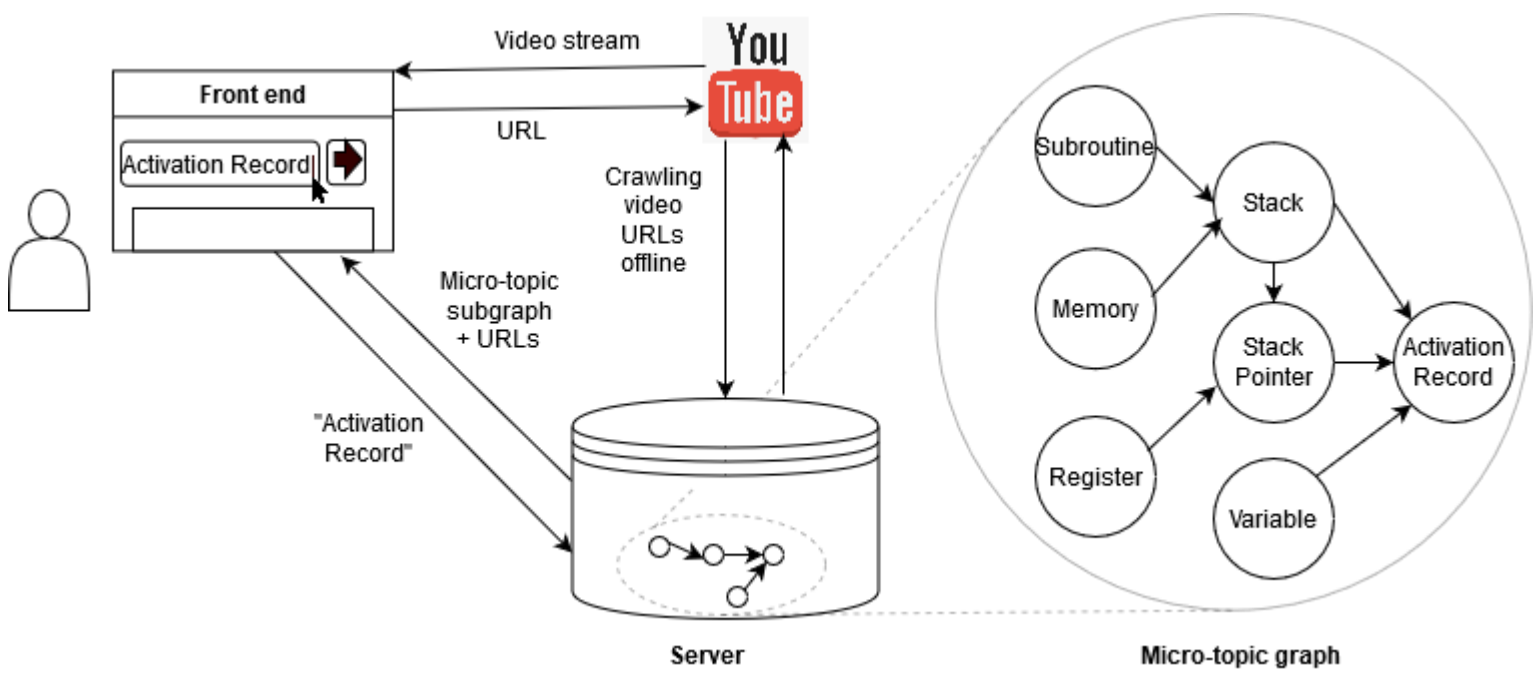

Figure 1. Architecture of the e-learning platform

E-learning platforms have become very popular over the last years since they allow individuals to acquire new skills. However, they all suffer from the lack of content adaptation because they address a topic by splitting it up into smaller blocks, to which we refer as micro-topics. Learners must then complete the list of learning materials in a fixed order, where the same set of learning materials is used for all learners. This does not consider the fact that individuals learn differently. In order to overcome this limitation, we are in the process of building an adaptive e-learning platform which provides different learning materials for micro-topics and which adjusts the order in which micro-topics will be covered. The order will only be constrained by prerequisites, i.e. if a micro-topic is required for understanding a more advanced one, it must be completed first. Since there could be arbitrarily many of such prerequisite constraints, we visualize them in a graph, to which we refer as micro-topic graph.

The client server architecture of our e-learning platform is depicted in Figure 1. The micro-topic graph, which serves as the key data structure, is stored on the server and is constructed offline. Initially, a set of micro-topics is automatically extracted from text documents such as books or slides according to (Wang et al., 2016). To understand in which order these micro-topics are to be processed by learners, we infer pairwise prerequisite relationships between the topics. This imposes a partial order on the micro-topic graph since directed edges are inserted between micro-topics if one is a prerequisite for another one. To compute these relationships, for the respective micro-topics, we crawled the subtitles of relevant YouTube videos and their corresponding URLs which are then stored in the database on the server side. The subtitles correspond to the text documents used for determining the pairwise prerequisites (Talukdar \& Cohen, 2012) using a method similar to (Liang et al., 2016). We refer to this graph as the micro-topic graph, in which learners may proceed with any micro-topic for which they satisfy all prerequisites. Given a micro-topic $m$, all the incoming edges of $m$ are coming from direct prerequisites.

All remaining operations are performed in real time. Whenever a learner wants to learn about a micro-topic, she submits a query through the front end, which is a mobile device in this case, and the query is then sent to the server for processing. The server queries the micro-topic graph to return the subgraph which contains the requested micro-topic and its prerequisites as a list of JSON objects, where each micro-topic contains additional metadata like URLs to multiple YouTube videos and which of those should be recommended to be watched first by the learner, i.e. their rankings.

Periodically, the server updates the URLs that should be recommended for a learner to initially watch based on her previous interactions with the e-learning platform. For example, as shown in Figure 1 in the sample micro-topic graph on the right-hand side, suppose a learner wants to learn about the micro-topic "Stack" and enters this subject in the search bar in the front end. Then the server will return the micro-topics "Subroutine", "Memory" and "Stack", where the former two are the only prerequisites for the latter. The query result will also include URLs to relevant YouTube videos for all three micro-topics, which were already stored on the 
server. For each of the micro-topics, multiple URLs for different learning materials are included which caters to the fact that individuals learn differently and might prefer different explanations. When the learner clicks on one of the returned micro-topics in the front end, the mobile device will open the respective URL to stream the video given that a learner satisfies all prerequisites to play this video. Part of the metadata for each micro-topic is also which of the video URLs should be displayed to a learner, but she is free to choose a different one.

YouTube as a video platform could be replaced by any other platform, but since it is the most popular one, we have decided to integrate it into our platform. We use YouTube videos instead of Wikipedia for inferring prerequisite relationships between micro-topics since some of them could be explained from different perspectives. Therefore, merging multiple related YouTube videos instead of using one Wikipedia entry yielded more robust results in our preliminary experiments. Another reason in favor of YouTube is that for some micro-concepts there are no entries in Wikipedia, while there are entries on YouTube.

\subsection{Exploration of Micro-Topics on Mobile Devices using a List Metaphor}

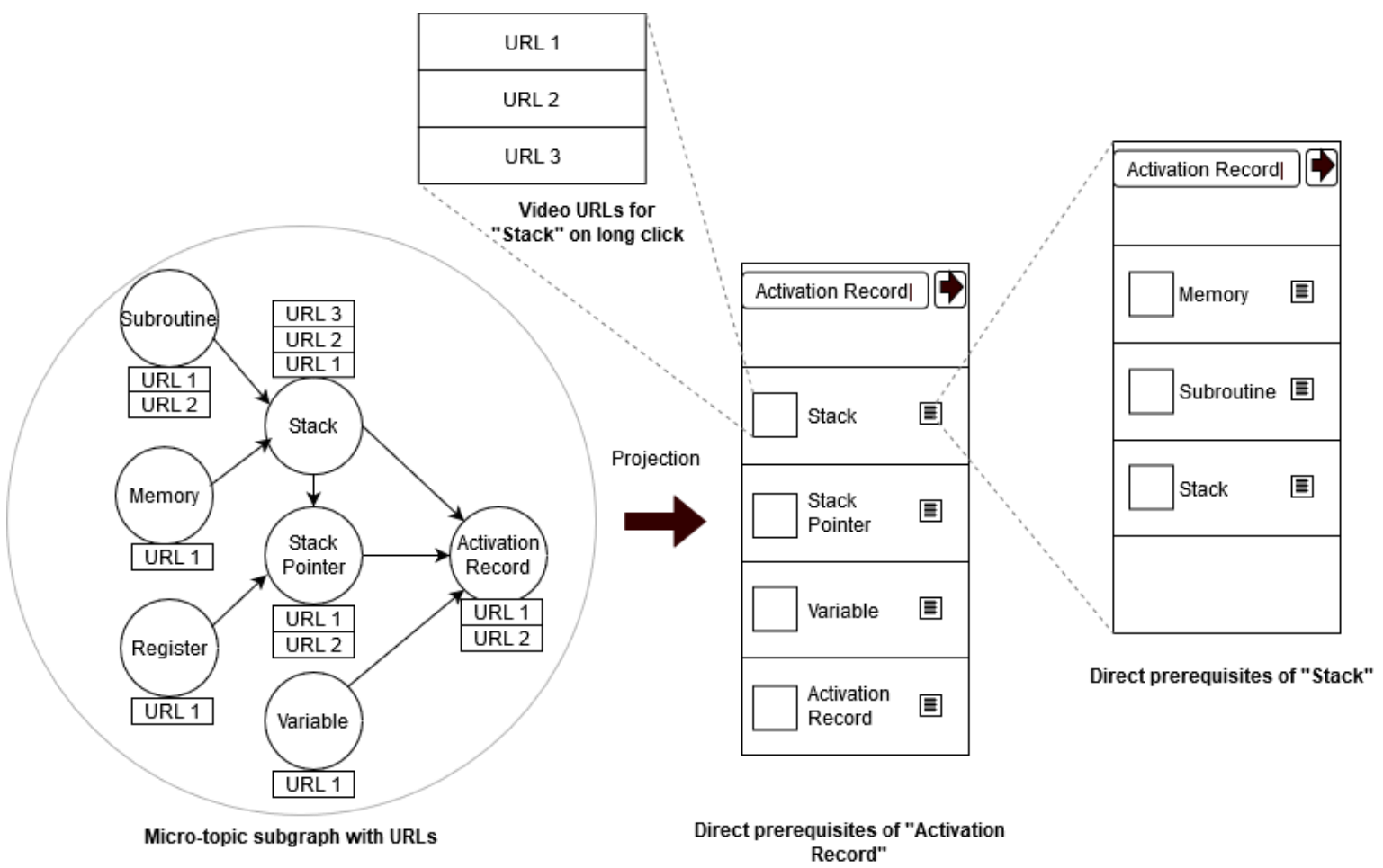

Figure 2. Mapping the micro-topic subgraph to the list metaphor on mobile devices

Given that the respective micro-topic subgraph, shown in Figure 2, was returned by the server for the topic "Activation Record" to the front end, the visualization of this subgraph depends on the type of front end. While computers have enough space to display an interactive graph, mobile devices, especially smartphones, lack it. Hence, a graph is not the best visual metaphor on mobile devices as it wastes space. Instead, we propose to use a metaphor based on lists which utilizes the limited space more effectively and avoids panning and zooming operations. This means that the micro-topic subgraph must be projected to this list according to Figure 2, where the desired micro-topic ("Activation record" in this case) is displayed at the bottom of the list and all direct prerequisites ("Stack", "Stack Pointer", "Variable") precede it in an arbitrary order as there cannot be a prerequisite relationship among direct prerequisites. This resembles a YouTube playlist where items at the top of the list will be played first. Long clicking on any micro-topic displays a list of available videos for that topic from which the learner may choose. 


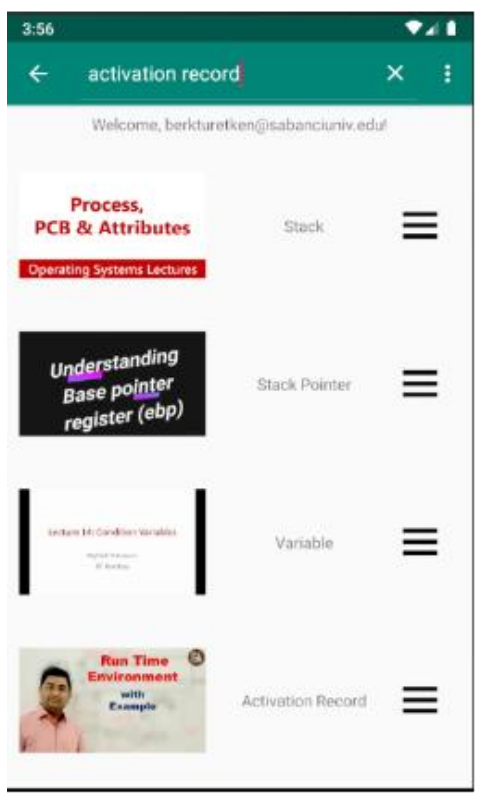

a)

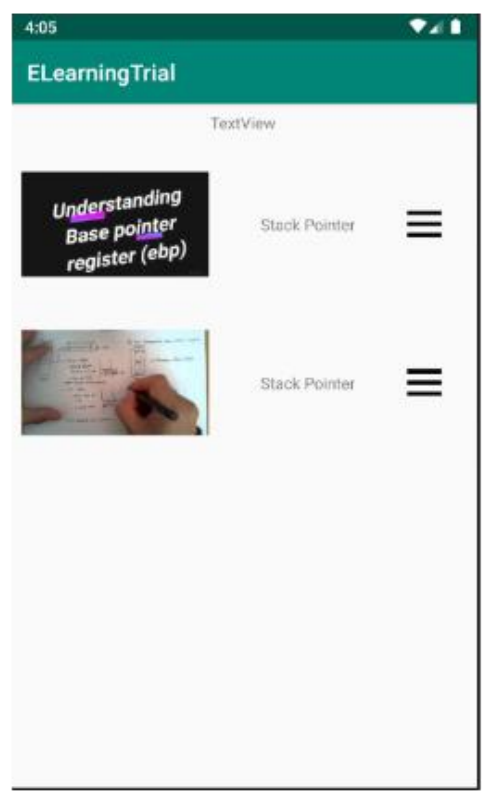

b)

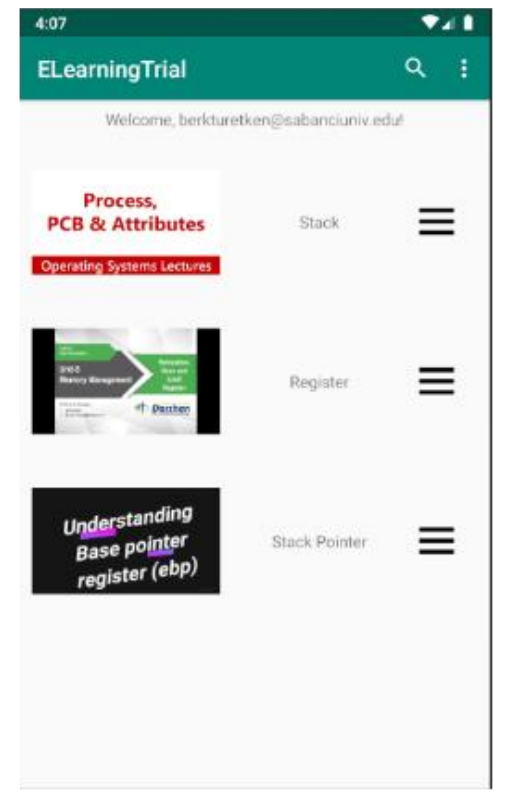

c)

Figure 3. Screenshots of the prototype. In a) the learner searched for "Activation Record". After long clicking on the video thumbnail of "Stack Pointer", a list of available videos for this micro-topic is displayed. In c) the learner clicked

in a) on the hamburger icon of "Stack Pointer" to see its direct prerequisites, which are "Stack" and "Register"

We implemented a prototype of our metaphor in Android as shown in Figure 3. Initially, a learner wants to learn about the micro-topic "Activation Record" from a course on operating systems (which makes it easier to follow our running example) and enters it in the search bar at the top. As a result of this query, the micro-topic subgraph with video URLs from Figure 2 is returned by the server and is visualized in Figure 3 a using our proposed list metaphor. For each micro-topic it displays a thumbnail of the most suitable video, that was determined by the server from the video URLs which were collected offline for that topic. The micro-topic the learner wants to complete, "Activation Record" is shown at the bottom of the list and the direct prerequisites "Stack", "Stack Pointer", and "Variable" must be finished prior to that which is indicated by them being ranked higher in the playlist. When long clicking on the video thumbnail of "Stack Pointer", the list with available videos for that topic pops up allowing the learner to select a different video. This is illustrated in Figure $3 \mathrm{~b}$. When clicking on the hamburger icon at the right hand side of "Stack Pointer", its direct prerequisites are displayed as a playlist with "Stack Pointer" being the last entry indicating that both its prerequisites, "Stack" and "Register", must be completed first. From this view they may return to Figure 3a at any time.

\section{PRELIMINARY EVALUATION RESULTS}

We performed a preliminary user study which assessed a) how well our proposed metaphor for mobile devices conveys the same information as a graph, b) how intuitive learners find the interactions, and c) any suggestions for improvement. Overall, 9 students from our university participated in this study and before starting the experiment, they were all given a short introduction about the available functionalities in the user interface and how they can be accessed. The information that was provided in that step will be explained by a tutorial in the actual mobile app but is not implemented yet. To address a), we asked them afterwards to perform three tasks that cover the different aspects of graphs, namely in which order a learner should watch micro-topics given the playlist (a.1), navigating the micro-topics through direct prerequisites (a.2), and changing learning materials (a.3). The original survey questions for the three tasks are listed below: 
a.1) Indicate in which order (from 1 to $n$, where $n$ is watched at the end) you would watch the videos when you searched for "Activation Record".

a.2) Which videos should you watch before watching the video about "Stack"? Please write the names down.

a.3) How many videos exist for the topic "Stack"? Please write down the number.

For b), participants rated our visual metaphor on a Likert scale from 1-5, where higher scores correspond to more positive feedback. After a) and b) participants suggested improvements for the user interface in the survey. Successful solutions to a.1), a.2), and a.3) were awarded with a score of 1 if participants used Any successful solution of a task from a) was awarded with a score of 1 , while a correct solution using a different way to achieve the correct result was counted as 0.5 ; otherwise it was counted as 0 . With 9 participants, the scores for each of the three tasks in a) could be at most 9 . The results are depicted in Table 1.

Table 1. Success rate of the 9 volunteers to solve the different tasks using the list metaphor

\begin{tabular}{lll}
\hline Task number & Task goal & Score \\
\hline a.1 & Determine micro-topic order & $9 / 9$ \\
a.2 & Micro-topic navigation & $9 / 9$ \\
a.3 & Changing learning materials & $4.5 / 9$
\end{tabular}

While participants were successful in navigating through the micro-topics and in understanding in which order to watch the videos, they encountered problems when having to select a different video for micro-topic. Roughly half of the participants failed to complete this task correctly.

For b), our metaphor was generally perceived positively which is reflected in an average score of 3.9 (out of 5). In terms of c), the lower score for changing learning materials was reflected in the comments we received. Participants shared different ideas on how to make this task more intuitive. The most frequently suggested improvement was replacing long click events by swipe actions. Other suggestions involved adding more visual feedback for completed videos with progress bars like in YouTube.

\section{CONCLUSION}

In this paper we have introduced a novel visual metaphor based on lists which allows learners to explore different micro-topics and their prerequisites. By focusing on the visualization of direct prerequisites of a micro-topic only, the small screen size is utilized effectively, and learners will not get overwhelmed with information even if there are hundreds of thousands of micro-topics in the graph. Moreover, no panning or zooming interactions are required to navigate through the micro-topics as opposed to a graph. However, as we stated before, our e-learning platform is still under development and the same holds for our mobile application. The metaphor needs to be refined to convey additional information such as which micro-topics learners had already completed in the past, which micro-topics are not accessible yet due to unsatisfied prerequisites, etc. The feedback from the preliminary study will also be incorporated. Once this is implemented, we will conduct a more extensive user study to evaluate if the positive feedback from our preliminary study for our proposed visual metaphor is confirmed.

\section{ACKNOWLEDGEMENT}

The authors would like to thank all participants of the preliminary user study for their constructive feedback to improve the user interface. 


\section{REFERENCES}

Buchem, I., \& Hamelmann, H. (2010). Microlearning: a strategy for ongoing professional development. eLearning Papers, 21(7), 1-15.

Cecilio-Fernandes, D., Cnossen, F., Jaarsma, D. A., \& Tio, R. A. (2018). Avoiding surgical skill decay: a systematic review on the spacing of training sessions. Journal of surgical education, 75(2), 471-480.

Drochtert, D., \& Geiger, C. (2015). Collaborative magic lens graph exploration. In SIGGRAPH Asia 2015 Mobile Graphics and Interactive Applications (pp. 1-3).

Du, F., Cao, N., Lin, Y. R., Xu, P., \& Tong, H. (2017, May). isphere: Focus+ context sphere visualization for interactive large graph exploration. In Proceedings of the 2017 CHI Conference on Human Factors in Computing Systems (pp. 2916-2927).

Kister, U., Klamka, K., Tominski, C., \& Dachselt, R. (2017, June). GraSp: Combining Spatially-aware Mobile Devices and a Display Wall for Graph Visualization and Interaction. In Computer Graphics Forum (Vol. 36, No. 3, pp. 503-514).

Liang, C., Wu, Z., Huang, W., \& Giles, C. L. (2015, September). Measuring prerequisite relations among concepts. In Proceedings of the 2015 conference on empirical methods in natural language processing (pp. 1668-1674).

Talukdar, P. P., \& Cohen, W. W. (2012, June). Crowdsourced comprehension: predicting prerequisite structure in wikipedia. In Proceedings of the Seventh Workshop on Building Educational Applications Using NLP (pp. 307-315). Association for Computational Linguistics.

Wang, S., Ororbia, A., Wu, Z., Williams, K., Liang, C., Pursel, B., \& Giles, C. L. (2016, October). Using prerequisites to extract concept maps from textbooks. In Proceedings of the 25th acm international on conference on information and knowledge management (pp. 317-326) 